\title{
Mixed Reality Neurosurgical Microscope for Training and Intra-operative Purposes
}

\author{
Alessandro De Mauro ${ }^{1}$, Joerg Raczkowsky ${ }^{1}$, Marc Eric Halatsch ${ }^{2}$, and Heinz Wörn ${ }^{1}$ \\ ${ }^{1}$ Institute for Process Control and Robotics, University of Karlsruhe (TH), Germany \\ ${ }^{2}$ Department of Neurosurgery, University Hospital of Heidelberg, Germany \\ demauro@ira.uka.de
}

\begin{abstract}
In recent years, neurosurgery has been deeply influenced by new technologies. It requires fine techniques targeted to obtain treatments minimally invasive though often traumatic. The precision of the surgical gesture is related both to experience of the surgeon and accuracy of the available technological instruments. Computer Aided Surgery (CAS) can offer several benefits for the patient's safety. From a technological point of view we observe the use of the Virtual Reality (VR) for the surgeons training and Augmented Reality (AR) for the intra-operative aid for treatments. This paper presents a prototype for a mixed reality system for neurosurgical interventions embedded on a real surgical microscope for pre- and intra- operative purposes. Its main purposes are: the realistic simulation (visual and haptic) of the spatula palpation of low-grade glioma and also the stereoscopic visualization in AR of relevant 3D data for safe surgical movements in the image guided interventions.
\end{abstract}

Keywords: virtual and augmented reality, physical modeling, haptic feedback, training systems, neurosurgery.

\section{Motivation and Medical Background}

In recent years, neurosurgery has been deeply influenced by new technologies. It requires fine techniques targeted to obtain treatments minimally invasive and traumatic. Intra-operative false movements can be devastating, leaving patients paralyzed, comatose or dead. The precision of the surgical gesture is related both to experience of the surgeon and accuracy of the available technological instruments. Computer Aided Surgery (CAS) can offer several benefits for the patient safety. Traditional techniques for training in surgery include the use of animals, phantoms and cadavers. The main limitation of these approaches is that live tissue has different properties from dead tissue and also that animal anatomy is significantly different from the human. Nowadays, this classical training is improved by the use of well illustrated books and excellent training movies recorded directly in the Operating Room (OR) but the main training for surgeons is still performed on the real patient. It is shown [1] that virtual reality simulators can speed-up the learning process and improve the proficiency of surgeons prior to performing surgery on a real patient.

Low-grade gliomas are intrinsic brain tumors that typically occur in younger adults. The objective of related surgery is to remove as much of the tumor as possible while 
minimizing damage to the normal brain. One of the obstacles associated with the surgical resection of these tumors is that the pathological tissue may closely resemble normal brain parenchyma when looked at through the neurosurgical microscope.

As a result, efforts to remove all tumor cells inevitably remove some of the normal brain and can leave behind small sections of tumorous cells. The remaining glioma cells continue to grow, eventually causing additional damage to the remaining normal brain, and a recurrence of symptoms.

Neuro-navigation can help only partially because the brain shift phenomena effects the pre-operative patient data after craniotomy and tissue removal. The tactile appreciation of this difference, in consistency of the tumor compared to normal brain, requires considerable experience on the part of the neurosurgeon.

These previous considerations are the main reasons to develop a neurosurgical training system but this is only a preoperative task. As described later, microscope, surgical tools and patient data are commonly used in the OR during the image guided operation. The hardware (microscope, tracking system, tools) and the software (navigation system based on the patient dataset) are both involved in the training and intraoperative activities. These considerations justify the idea of a mixed reality system that uses similar environment and architecture setups for pre- and intra-operative use, in a natural continuum training system (based on VR) and in an intra-operative system (based on AR).

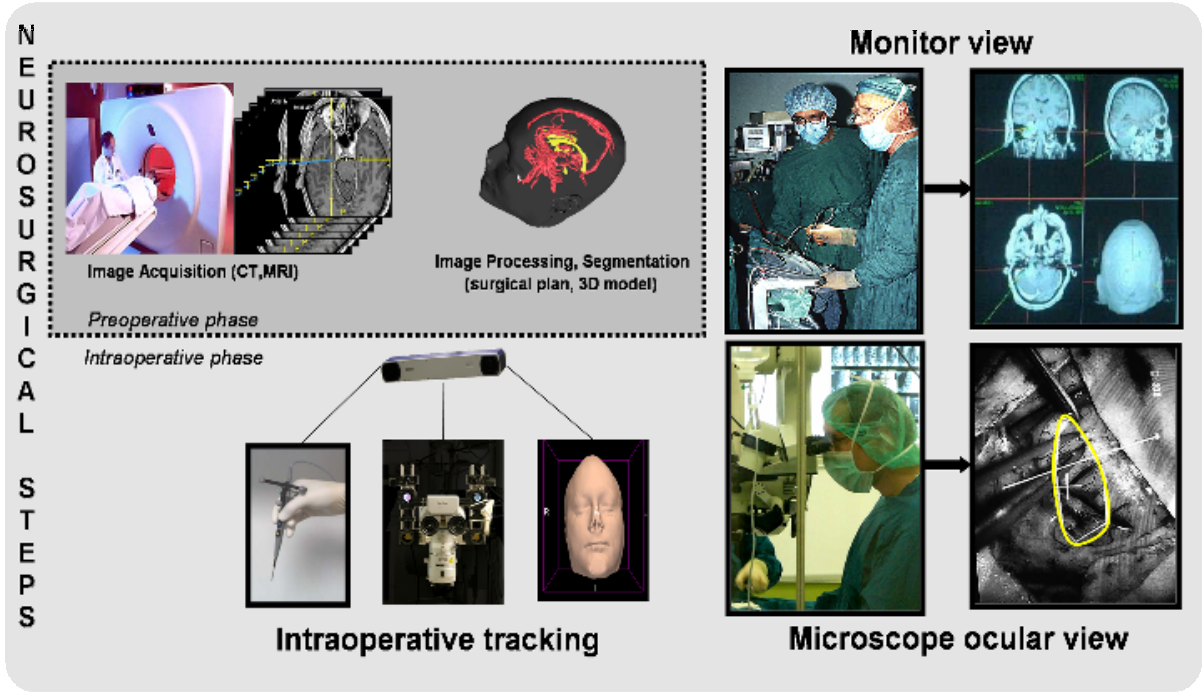

Fig. 1. Common steps in neurosurgery. Patient images are acquired and processed. 3D model of the region of interest are obtained. In the intra-operative phase this data are available on the screen and used for the navigation.

\section{Neurosurgical Workflow}

At this point, it is particularly important to give briefly an introduction about all the steps involved before and during the neurosurgical procedures. The geometric models 
of the organs or the region of interest (e.g. tumor) are reconstructed from data acquired by CT, MRI or other means by a radiologist. In the intra-operative phase a tracking system is used to track the relative positions of the patient, relevant tools, and microscope. All data are shown on the video using the normal three views (coronal, axial, sagittal). In the OR, a surgeon's eyes are typically on the microscope oculars but occasionally they need to see the screen in order to understand the correct position compared to the preoperative images (CT, MRI). The position and orientation of an active tool tracked by the infrared tracking system and it's relative position in the patient images are shown on the monitors. The two-dimensional contour of the region of interest is recognized as defined by the radiologist in the preoperative step. This two-dimensional shape is visible inside the commercial microscopes overlaid to the oculars views. The steps discussed here are shown in Fig.1. Nowadays, the threedimensional environment reconstruction from two dimensions is another difficult and critical mental work for the surgeon.

\section{State of the Art in Training Systems}

The success of using haptic devices in medical training simulators has already been demonstrated by several commercial companies working in this field (Immersion Medical [3], Surgical Science [4], Mentice [5], and Reachin Technologies[6], for example) and in other research works [7, 8, 9, 10, 11, 12 and 13].

Webster et al. [14] present a haptic simulation environment for laparoscopic cholecystectomy, and Montgomery et al. [15] shows a simulation environment for laparoscopic hysteroscopy; both projects focus on haptic interaction with deformable tissue. Cotin et al. [16] present a haptic simulator for interventional cardiology procedures, incorporating blood flow models and models of cardiopulmonary physiology. De et al. [17] apply the method of finite spheres to a haptic simulator for laparoscopic GI surgery.

On the other hand, the state-of-the-art in neurosurgical simulation [18] shows only a few examples of VR based systems which use force feedback [19, 20, and 21]. Because a neurosurgical microscope is used in a large percentage of interventions, the realism of these simulators is limited by the architecture: they use either standard monitors or head mounted displays but not a real surgical microscope.

\section{Methods and Tools for a Virtual Training}

In neurosurgical interventions monitors and operating microscopes are both commonly employed. In order to understand the correct tumor position compared to the preoperative images (CT, MRI) a surgeon's eyes are normally on the microscope oculars and only occasionally glance at a larger screen. This second view is very important in image guided therapy to check the correct position of the surgical tools inside the patient brain. A complete simulation system for neurosurgical training requires:

- simulation of the virtual patient inside microscope oculars;

- force feedback rendering directly at the user's hand;

- navigation software actually used in OR (i.e. BrainLab or Stryker). 


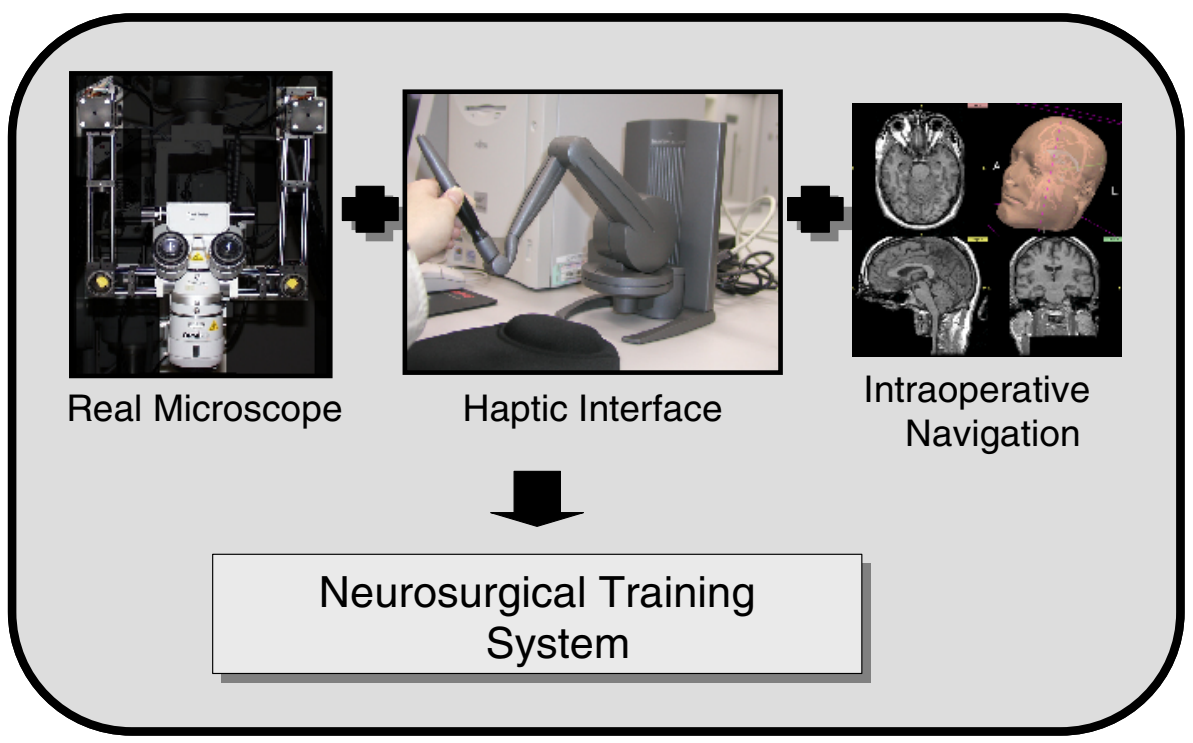

Fig. 2. Left: simulator concepts. Left: ocular views of the deformations of the $3 \mathrm{D}$ patient reconstructed from patient pre-operative images.

A virtual environment is geometrically built using real patients' data affected by a low grade glioma from the standard medical imaging devices. Human organs are accurately reconstructed from real patient images using the software package 3DSlicer [22]. A region growing algorithm has been used for segmentation, and next organ and region of interests (ROI) are classified. The 3D organ data obtained is imported directly into our application and the registration step between these two virtual environments is carried out. 3D models of the surgical tools are provided using a Laser ScanArm (FARO) [23].

The visual output is provided by the rendering software developed in $\mathrm{C}++$ and built on the open source GPU licensed and cross-platform H3D [24] (version 2.0), a scenegraph API based on OpenGL is used for graphics rendering.

Collision detection modules based on the physical model for the simulation of tool interactions and brain deformations are developed on OpenGL to obtain high level performance and realism. Hierarchies of bounding volumes are used to perform fast collision detection between complex models, with the employed method based on model partition [25], the strategy of subdividing a set of objects into geometrically coherent subsets and computing a bounding volume for each subset of objects. The physical modeling method is based on mass-spring-damper (MSD) and consists of a mesh of point masses connected by elastic links and mapped onto the geometric representation of the virtual object. This method is employed in our prototype to describe the mechanical properties of the virtual bodies computing the force feedback to the haptics and the organ deformations to be visualised. It's a discrete method characterized by low computable load, simplicity, low accuracy and low risk of instability because it uses Newton dynamics to modify the point-masses positions and creates deformations with consideration to volume conservation. Brain tissue properties are 
modeled with MSD upon OpenHaptics library (Sensable) [26]. The tissue parameters are evaluating together with our medical partner (Department of Neurosurgery, University Hospital of Heidelberg) using different training sections and processing empiric data.

Different haptic renderings were tested for a better optimization of the deformations. In order to have a complete training platform a video navigation system containing different 3D volume views is required. To achieve this, we have connected our system with the image guided therapy module of 3DSlicer. We are using a haptic device (Phantom Desktop) in order to provide the surgeon with an immersive experience during the interaction between the surgical tools and the brain or skull structures of the virtual patients. The force feedback workspace and other important properties (nominal position resolution and stiffness range) make it suitable to be used in an ergonomic manner in conjunction with the microscope.

The 3D real-time position of the two viewpoints (one of each microscope ocular) is determined in the virtual environment through the use of passive markers affixed to the microscope and tracked by the infrared optical tracker (Polaris NDI). In order to speed up the simulation we modified the original library for tracking developed in our laboratories. In this way, data collected from Polaris is sent using the local area network to several given IP addresses and ports using the open-source OpenIGTLink of 3DSlicer modified for our needs. This allows a distributed architecture with the separation between rendering (graphical and haptic) and tracking PC with advantages in terms of computational load and realism (the average frame rate for graphical rendering is $31 \mathrm{fps}$ and for the haptic $998 \mathrm{fps}$ ). The collisions between organs and surgical tools produce forces which have to be replicated by the haptic interface and organ deformations, which have to be graphically rendered.

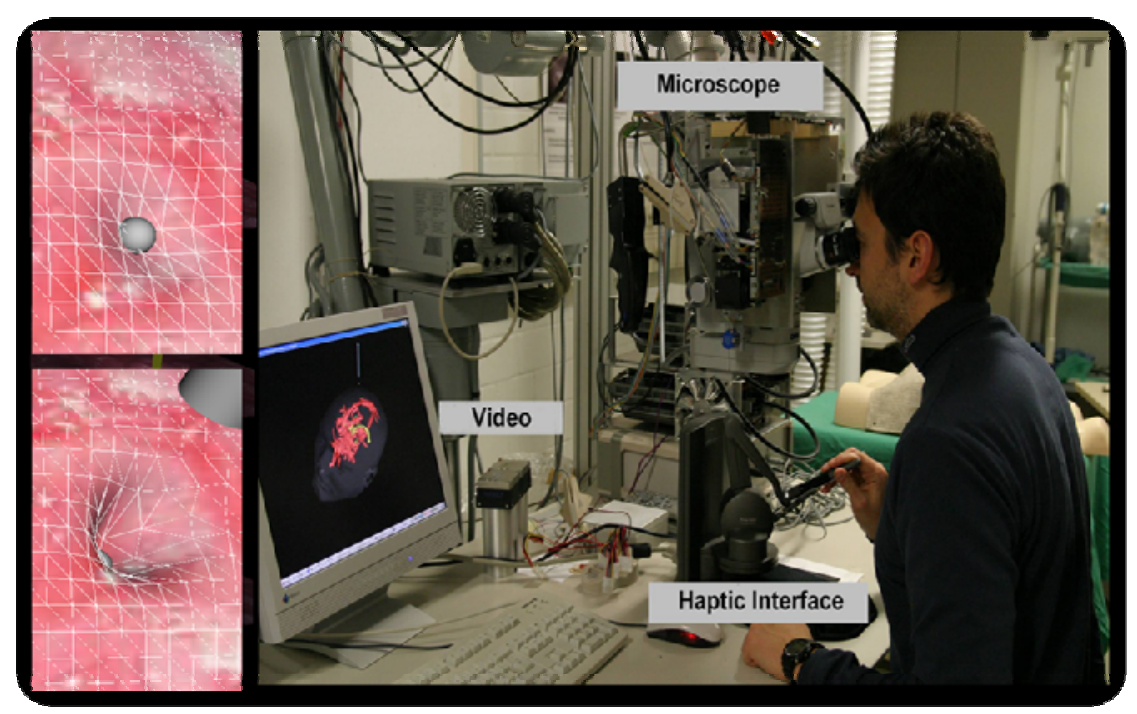

Fig. 3. Simulator. Left: brain tissue deformations. Right: complete prototype. 
All the basic surgical operations on the brain are suitable to increase surgery skills in a fast and safe way using virtual reality. The main operating task simulated is the visual and tactile sensations of brain palpation (healthy or affected by low grade glioma) pushing aside the human tissue using a neurosurgical spatula.

The 3D environment description language used is $\mathrm{X} 3 \mathrm{D}$, which is the natural successor of the well known VRML. It means that the complete scene in the application can be rendered in a web browser for future extension as web- collaborative and distributed training.

\section{Extension to Intra-operative Augmented Reality}

The architecture described can be used for intra-operative purposes. In this instance, a surgeon needs to use the microscope, monitors and surgical tools. This is the basic setup for an image guided therapy interventions. The same virtual environment can be AR rendered in to the microscope optics with the difference that now the complete anatomy is considered rigid (deformations are not requested in this step).

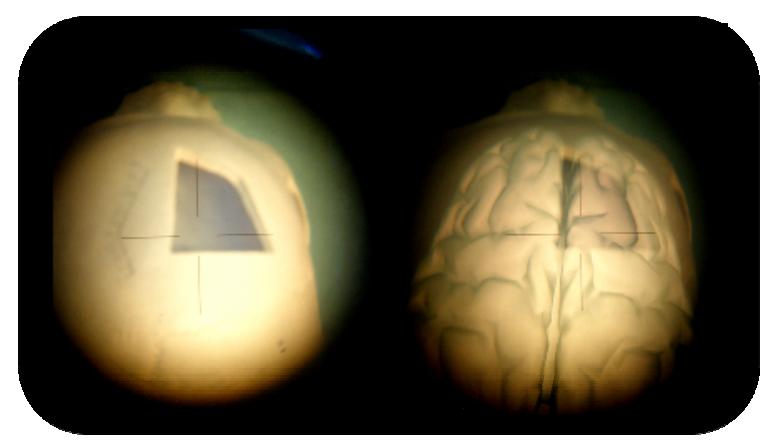

Fig. 4. Augmented reality microscope. Left: skull. Right: 3D model of the brain superimposed on the skull.

The haptic interface is no longer required and is replaced by new navigated infrared active tools.

The prototype is capable of tracking, in real time, the microscope, the patient's head and one surgical tool (pointer with active markers).

Fig. 4 shows the augmented reality views inside the microscope oculars in which is possible identify 3D region of interest (in this example the brain surface is rendered). The microscope hardware related part was realized at our institute and described in a previous work [27]. Registration and camera calibration are required for a perfect alignment between real and virtual world. Both are off-line steps with similar approaches used in [27]. 


\section{Conclusions}

This paper presents the development of the first mixed reality system for training and intra-operative purposes in neurosurgery embedded on a real microscope. The main training task is the advanced simulation of brain tissue palpation enabling the surgeon to distinguish between normal brain tissue, and that tissue affected with a low grade glioma. Force feedback interaction with soft and hard tissues is provided to the surgeon's hands in order to provide a complete immersive experience.

The second feature allows the system to be employed as an augmented reality microscope inside the OR. In this case a complex 3D environment is rendered by a stereoscopic image injection directly inside the microscope oculars for a better real time brain navigation and space cognition. 3DSlicer is, in both previous functional modalities, directly connected to the NDI tracking system in order to provide the navigation inside the real patient's images on the screen. The previously described software architecture guarantees performances and portability.

The architecture and the main features were defined in tight collaboration with surgeon staff and we are evaluating other improvements in providing more specific simulation tasks extending this work.

All the components are open source or at least based on a GPL license.

\section{Acknowledgments}

This research is a part of the European project "CompuSurge" funded by EST "Marie Curie" research network.

\section{References}

1. Ro, C.Y., et al.: The LapSim. A learning environment for both experts and novices. Department of Surgery, St. Luke's-Roosevelt Hospital Center and Columbia University, New York, USA (2005)

2. Hill, D.L.G., Maurer Jr., C.R., Martin, A.J., Sabanathan, S., Hall, W.A., Hawkes, D.J., Rueckert, D., Truwit, C.L.: Assessment of intraoperative brain deformation using interventional MR imaging. In: Taylor, C., Colchester, A. (eds.) MICCAI 1999. LNCS, vol. 1679, pp. 910-919. Springer, Heidelberg (1999)

3. Immersion (status January 2008), http: / / www. immersion. com/

4. SurgicalScience (status January 2008), http: / /www. surgical-science.com/

5. Mentice (status January 2008), http: / /www . mentice.com/

6. Reachin (status January 2008), http: / /www . reachin. se/

7. Basdogan, C., Ho, C., Srinivasan, M.A.: Virtual Environments for Medical Training: Graphical and Haptic Simulation of Common Bile Duct Exploration. IEEE-ASME Trans. Mechatronics 6(3), 26-285 (2001)

8. Çakmak, H.K., Kühnapfel, U.: Animation and Simulation Techniques for VR-Training Systems in Endoscopic Surgery. In: Proc. Eurographics Workshop Animation and Simulation (EGCAS 2000), pp. 173-185 (2000)

9. Brown, J., et al.: Algorithmic Tools for Real-Time Microsurgery Simulation. Medical Image Analysis 6(3), 289-300 (2002) 
10. Cotin, S., Delingette, H., Ayache, H.: Real-Time Elastic Deformations of Soft Tissues for Surgery Simulation. IEEE Trans. Visualization and Computer Graphics 5(1), 62-73 (1999)

11. Wu, X., et al.: Adaptive Nonlinear Finite Elements for Deformable Body Simulation Using Dynamic Progressive Meshes. In: Chalmers, A., Rhyne, T.-M. (eds.) Proc. Eurographics, Computer Graphics Forum, vol. 20(3), pp. 349-358 (2001)

12. Székely, G., Brechbühler, C., Hutter, R., Rhomberg, A., Schmid, P.: Modelling of Soft Tissue Deformation for Laparoscopic Surgery Simulation. In: Wells, W.M., Colchester, A.C.F., Delp, S.L. (eds.) MICCAI 1998. LNCS, vol. 1496, pp. 550-561. Springer, Heidelberg (1998)

13. O'Toole, R., et al.: Measuring and Developing Suturing Technique with a Virtual Reality Surgical Simulator. J. Am. College of Surgery 189, 114-128 (1999)

14. Webster, R., Haluck, R.S., Zoppeti, G., et al.: A haptic surgical simulator for laparoscopic cholecystectomy using real-time deformable organs. In: Proc. IASTED International Conference on Biomedical Engineering, Salzburg, Austria, June 25-27 (2003)

15. Montgomery, K., et al.: Surgical simulator for operative hysteroscopy. In: IEEE Visualization 2001, pp. 14-17 (2001)

16. Cotin, S., Dawson, S.L., Meglan, D., Shaffer, D.W., Farrell, M.A., Bardsley, R.S., Morgan, F.M., Nagano, T., Nikom, J., Sherman, P., Walterman, M.T., Wendlandt, J.: ICTS: An Interventional Cardiology Training System. In: Proceedings of Medicine Meets Virtual Reality, pp. 59-65 (2000)

17. De, S., Kim, J., Manivannan, M., Srinivasan, M.A., Rattner, D.: Multimodal Simulation of Laparoscopic Heller Myotomy Using a Meshless Technique. In: Proceedings of Medicine Meets Virtual Reality (MMVR), Newport Beach, pp. 127-132 (2002)

18. Goh, K.Y.C.: Virtual reality application in neurosurgery. In: Proceedings of the 2005 IEEE, Engineering in Medicine and Biology 27th Annual Conference, Shanghai (2005)

19. Luciano, C., Banerjee, P., Lemole, M.G., Charbel, F.: Second generation haptic ventriculostomy simulator using the immersivetouch ${ }^{\mathrm{TM}}$ system. In: Proceedings of Medicine Meets Virtual Reality 14, Long Beach, CA (2008)

20. Sato, D., Kobayashi, R., Kobayashi, A., Fujino, S., Uchiyama, M.: Soft tissue pushing operation using a haptic interface for simulation of brain tumor resection. Journal of robotics and mechatronics 18, 634-642 (2006)

21. Wiet, G., Bryan, J., Sessanna, D., Streadney, D., Schmalbrock, P., Welling, B.: Virtual temporal bone dissection simulation. In: Westwood, J.D. (ed.) Medicine Meets Virtual Reality 2000, Amsterdam, The Netherlands, pp. 378-384 (2000)

22. 3DSlicer (status January 2008), http: / /www. slicer. org/

23. Faro (status January 2008), http: / / www . faro. com

24. H3D (status January 2008), http: / /www . h3 dapi . org/

25. Van den Bergen, C.: Collision detection in interactive 3D environment. Elsevier Morgan Kaufmann, S. Francisco, USA (2004)

26. OpenHaptics (status March 2009), http: //www. sensable.com/products-openhaptics-toolkit.htm

27. Aschke, M., et al.: Augmented reality in operating microscopes for neurosurgical interventions. In: Wolf, Strock (eds.) Proceedings of 1st International IEEE EMBS Conference on Neural Engineering, pp. 652-655 (2003) 